PrePared For SUbMission to JINST

$8^{\text {th }}$ International Workshop on Semicondactor Pixel Detectors for Particles and IMAGING

5-9 SePtember 2016

SESTRI LeVANTEWHERE, ITALY

\title{
Physics performance of the ATLAS Pixel Detector
}

\section{S. Tsuno ${ }^{a, 1}$ on behalf of the ATLAS Collaboration}

${ }^{a}$ High Energy Accelerator Research Organization, KEK,

1-1 Oho, Tsukuba, Ibaraki 305-0801, Japan

E-mail: Soshi.Tsuno@cern.ch

Abstract: One noticeable upgrade from Run-1 to Run-2 with ATLAS detector in proton-proton collisions at LHC is the introduction of the new pixel detector, IBL, located near the beam pipe as the extra innermost pixel layer. The tracking and vertex reconstruction are significantly improved and good performance is expected in high level object such a $b$-quark jet tagging, in turn, it leads the better physics results. This note summarizes what is the impact on the IBL detector to the physics results especially focusing on the analyses using the $b$-quark jets throughout 2016 summer physics program.

KEYwords: ATLAS, pixel

\footnotetext{
${ }^{1}$ Corresponding author.
} 


\section{Contents}

1 Introduction 1

2 Application to the physics analysis 2

3 Physics performance 3

3.1 Search for SUSY in events with multiple $b$-quark jets 3

3.2 Search for the Standard Model Higgs association with top-quark pair production 4

3.3 Search for long-lived SUSY particles using pixel dE/dx 5

4 Summary 5

\section{Introduction}

This year, summer 2016, the ATLAS collaboration [1] published 54 conference papers and 3 supporting documents [2]. Among them, about $65 \%$ of the physics results uses the $b$-quark jet directly or indirectly in their analyses. Figure 1 summarizes the physics papers harvested in this year (summer 2016) categorized by the $b$-quark jet multiplicity.

There are 5 papers that use more than $3 b$-quark jets in the analysis. They search for SUSY particles that decay into top or bottom squarks followed by multi-top final state. The searches for the Standard Model Higgs boson associated with the top-quark pair production and double Higgs boson production mode are also in this category. When a heavy particle decays into leptons, the requirement of $b$-quark jet multiplicity is loosened to one or two $b$-quark jets. The other category is to use the $b$-quark jet indirectly. If the top-quark pair production is a dominant background, it is used to veto an event with $b$-quark jet to suppress, or to require $b$-quark jet explicitly to control such type of backgrounds. The analyses that do not use the $b$-quark jet at all target rather simple physics topologies like dilepton, dijet and $\gamma \gamma$ resonance or multi-lepton final state searches.

One of major upgrade in the ATLAS detector is the introduction of the new pixel detector, Insertable B-Layer (IBL) [3], near the beam pipe as the extra innermost pixel layer. The original design of the pixel detector [4] was three barrel layers and three disks system on each detector side and operated through Run-1 in 2010-2012 recorded luminosity of $\sim 20 \mathrm{fb}^{-1}$ of $\sqrt{s}=7,8 \mathrm{TeV}$ protonproton collision events. During the long-shutdown in 2013-2014, the IBL was newly constructed and installed. The Run-2 experiments started in 2015 with increased energy of $\sqrt{s}=13 \mathrm{TeV}$. The system now becomes a four-barrel layer system in Run-2. The former pixel detector has about $80 \mathrm{M}$ pixel channels with $50 \mu \mathrm{m} \times 400 \mu \mathrm{m}$ pitch in azimuth and $\mathrm{z}$ side, while the IBL adds another $12 \mathrm{M}$ pixel channels with $50 \mu \mathrm{m} \times 250 \mu \mathrm{m}$ pitch size. In IBL, two different sensor technologies are used; the planar sensor used in $75 \%$ of the total IBL volume that covers in the middle of the barrel region, and 3D sensor technology in the rest. 
Together with the increased colliding beam energy from $8 \mathrm{TeV}$ to $13 \mathrm{TeV}$, the installation of such a new detector will bring significant improvements in the physics analyses. This note highlights the physics performance obtained in Run-2 dataset and discusses the impact with IBL detector.

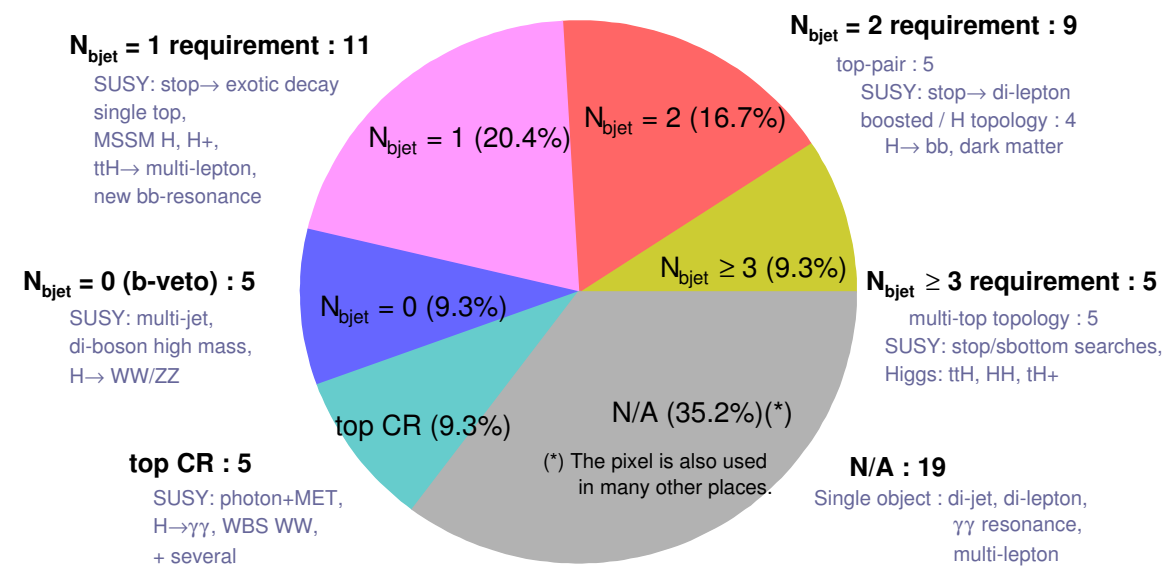

Figure 1. Summary of the physics papers obtained this year (summer 2016) categorized by the $b$-quark jet multiplicity [2].

\section{Application to the physics analysis}

The pixel detector is primary used in the charged particle track reconstruction. The pixel detector is the most sensitive device to determine the interaction points in $p p$ collision events, where about 23 multiple interactions on average (pileup) occur in one bunch beam crossing in an event in 2016. To discriminate the interesting interaction point from the pileup interactions, a good separation power is mandatory.

Figure 2 shows the impact parameter resolution of the tracks from Run-1 (without IBL) and Run-2 (with IBL) as a function of track $p_{T}$ [5] (a) and expected performance of the $b$-quark jet tagging algorithm [6] (b). The impact parameter resolution is largely improved in low $p_{T}$ tracks and almost factor 5 better rejection against light-flavor jets is achieved in the $b$-quark jet tagging algorithm. In addition, it is the first time that the Neural Network-based cluster-separation algorithm [7] is deployed to solve the shared cluster formed by the multiple particles into individual clusters. The algorithm works in the dense environment of the particle multiplicity in high $p_{T}$ jets, and recovers track reconstruction efficiencies without ambiguities.

The typical order of the $b$-quark jet identification uncertainties in the physics analyses is summarized in Table 1 as a category of the $b$-quark jet multiplicity. The jet energy scale uncertainties (JES) are also listed. There is obvious correlation of the systematic uncertainties as increase of the $b$-quark jet multiplicity. Contrary, the JES uncertainty widely distributes across the $b$-quark jet multiplicity and the $b$-quark jet identification uncertainty becomes larger systematic source than JES uncertainty when high $b$-quark jet multiplicity is required. The pixel detector will play a key 


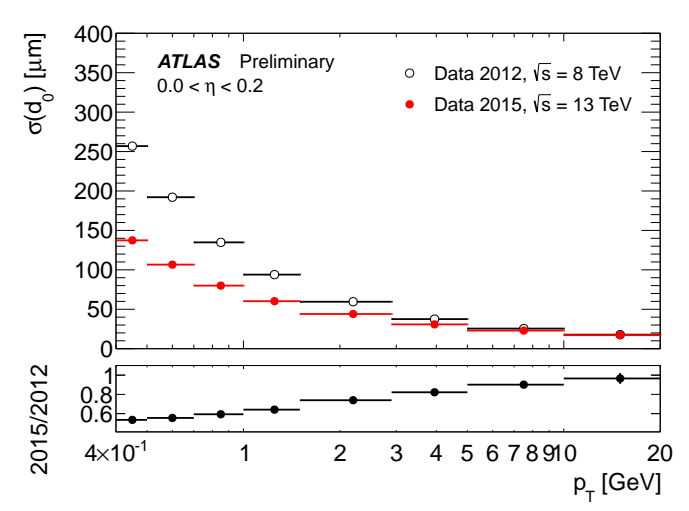

(a)

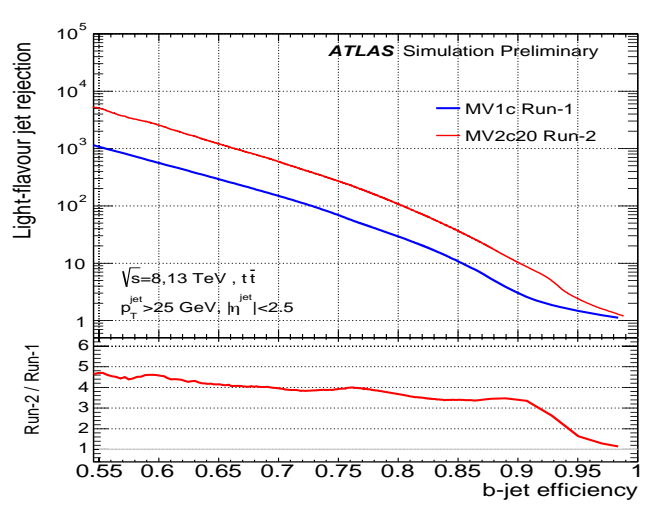

(b)

Figure 2. Impact parameter resolution of the tracking from Run-1 (without IBL) and Run-2 (with IBL) [5] (a) and expected improvement of the $b$-jet flavor tagging algorithm [6] (b).

rule to better control the $b$-quark jet identification performance. In the following section, individual physics analyses are discussed.

Table 1. Typical order of the $b$-quark jet identification uncertainties as a category of the $b$-quark jet multiplicity together with jet energy scale uncertainties (JES) used in the physics analyses for 2016 summer.

\begin{tabular}{|l|c|c|}
\hline Analysis & JES & $b$-jet \\
\hline$N_{b \text { jet }} \geq 3$ & $13-15 \%$ & $10-30 \%$ \\
$N_{b \text { jet }}=2$ & $4-10 \%$ & $1-15 \%$ \\
$N_{b \text { jet }}=1$ & $2-20 \%$ & $2-10 \%$ \\
$b$-veto & $1-20 \%$ & $\sim 3 \%$ \\
top CR & $12-33 \%$ & $7-16 \%$ \\
\hline
\end{tabular}

\section{Physics performance}

\subsection{Search for SUSY in events with multiple $b$-quark jets}

A search for supersymmetry involving the pair production of gluinos decaying into top or bottom squarks was carried out using dataset corresponds to an integrated luminosity of $14.8 \mathrm{fb}^{-1}$ with $\sqrt{\mathrm{s}}$ $=13 \mathrm{TeV} p p$ collisions [8]. The signal is searched for events with several high energetic jets, which contains at least three $b$-quark jets, large missing transverse momentum and isolated electrons or muons. No excess is found and upper limit on the production cross section was set to be $0.87 \mathrm{fb}$. The gluino masses less than $1.89 \mathrm{TeV}$ are also excluded at 95\% CL in simplified models of the pair production of gluinos decaying via sbottom or stop. The exclusion limits in the $\tilde{\chi}_{0}^{1}$ and gluino mass plane for the simplified model is presented in Fig. 3. This result significantly extends the exclusion limit obtained with the 2015 dataset [9], where the gluino mass limit was less than $1.35 \mathrm{TeV}$ at $95 \%$ CL with corresponding luminosity of $20.1 \mathrm{fb}^{-1}$.

The production cross section of such a heavy particle is roughly scaled by the parton luminosity and increased by a factor 8 times at new particle mass of $1.2 \mathrm{TeV}$ from $8 \mathrm{TeV}$ (Run-1) to $13 \mathrm{TeV}$ (Run-2) $p p$ collisions [10]. The luminosities used both in Run-1 and Run-2 datasets have about 
$30 \%$ difference but marginal order to be compared with the cross section differences. Given high mass search region, the backgrounds will not contribute since the enhancement of the SM processes from $8 \mathrm{TeV}$ to $13 \mathrm{TeV}$ is not so large. Hence the scaling of the signal cross section is a good measure to estimate the prospect of the exclusion limit if the Run-1 analysis without IBL is performed to the $13 \mathrm{TeV}$ dataset. This gives the gluino mass limit on $1.6 \mathrm{TeV}$ at $95 \% \mathrm{CL}$ and confirms the Run-2 prospect report [11]. Thus, it follows that the extension beyond $1.6 \mathrm{TeV}$ to $1.9 \mathrm{TeV}$ is purely due to the inclusion of the new detector IBL and improvement of the analysis itself in Run-2. In fact, the signal yield is much improved. The IBL obviously plays a key rule in the large extension of the sensitivity.

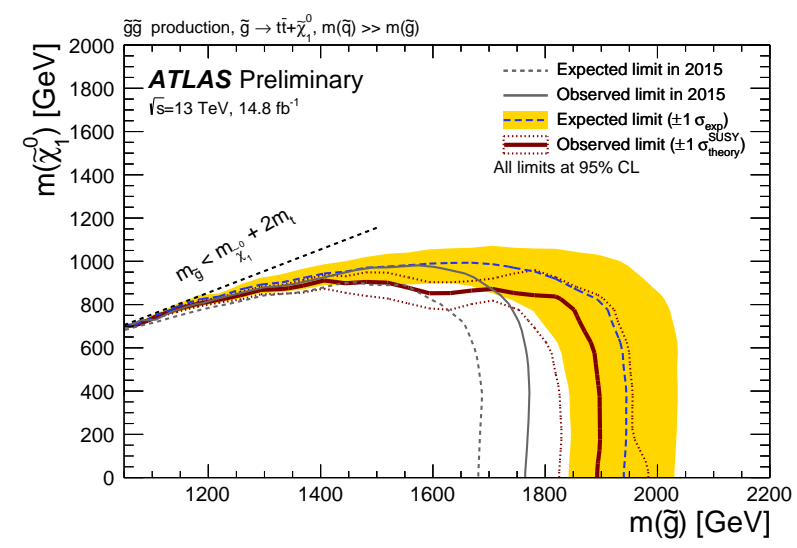

Figure 3. Exclusion limits in the $\tilde{\chi}_{0}^{1}$ and gluino mass plane for the simplified model [8]. The gluino masses less than $1.89 \mathrm{TeV}$ are excluded at $95 \% \mathrm{CL}$ in simplified models of the pair production of gluinos decaying via sbottom or stop, while $1.35 \mathrm{TeV}$ was achieved from Run-1 dataset. If the Run-1 analysis without IBL is performed to the $13 \mathrm{TeV}$ dataset, the limit of $1.6 \mathrm{TeV}$ is obtained by statistical scaling.

\subsection{Search for the Standard Model Higgs association with top-quark pair production}

A search for the Standard Model Higgs bosons produced in association with top-quark pair production is reported using $13.2 \mathrm{fb}$ of $p p$ collision dataset [12]. The production mode, $t \bar{t} H$ is still a missing channel which is not discovered yet and serve as the direct probe of the Yukawa coupling with the top-quark. The search is designed for the $H \rightarrow b \bar{b}$ decay mode and uses events with at least one top quark decaying to an electron or muon. The data are consistent with either the background-only hypothesis or with the Standard Model $t \bar{t} H$ prediction. The ratio of the measured $t \bar{t} H$ signal cross-section to the Standard Model expectation $(\mu)$ is found to be $\mu=1.6 \pm 1.0$ (syst.) \pm 0.5 (stat.) at a Higgs boson mass of $125 \mathrm{GeV}$ in the single lepton channel. This can be compared with the Run-1 result of $\mu=1.2 \pm 1.0$ (syst.) \pm 0.8 (stat.) [13].

The signal cross section is scaled by 3.9 from $8 \mathrm{TeV}$ to $13 \mathrm{TeV}$. But at the same time, the dominant background $t \bar{t}$ is also enhanced by same order, 3.3. Thus, the increased beam energy is not expected to enlarge the signal sensitivity. The systematic uncertainties are the same in both cases. The size of the statistical error however is almost $60 \%$ difference between Run-1 and Run- 2 . This can be interpreted as the pure gain of the signal yield in Run-2. As shown in Fig. 2, the rejection power of the light-flavor jets is significantly improved by a factor 5 in Run-2 at the $70 \% b$-quark 
jet tagging efficiency working point. Non-heavy flavor backgrounds are thus largely suppressed. This results in better signal to background ratio of $6.1 \%$ in Run-2, while it is $4.0 \%$ in Run- 1 . The difference of this signal to background ratio is same order with that in the statistical uncertainty of $\mu$, thanks to IBL and improved $b$-quark jet tagging performance in Run-2.

\subsection{Search for long-lived SUSY particles using pixel dE/dx}

A search for massive charged long-lived particles is presented using the dataset of $3.2 \mathrm{fb}^{-1}$ [14]. Many extensions of the Standard Model predict the existence of massive charged long-lived particles, such as R-hadrons. These massive particles are expected to be produced with a velocity significantly below the speed of light, and therefore to have a specific ionization higher than any Standard Model particle of unit charge at high momenta. The pixel detector is used to measure the ionization energy loss of reconstructed charged particles and to search for such highly ionizing particles. No deviation from the Standard Model background expectations is observed, and lifetime-dependent upper limits on R-hadron production cross-sections and masses are set to be above $0.4 \mathrm{~ns}$ of the lifetime of the gluino R-hadrons decaying to $q \bar{q}$ plus a $100 \mathrm{GeV}$ neutralino at the $95 \% \mathrm{CL}$, with lower mass limit ranging between $740 \mathrm{GeV}$ and $1590 \mathrm{GeV}$. The limit extends significantly than that in Run-1 [15]. The exclusion limits are presented both Run-1 and Run-2 results in Fig. 4. It is mentioned that the signal yield is enhanced by $15 \%$ due to the introduction of the IBL in Run- 2 .

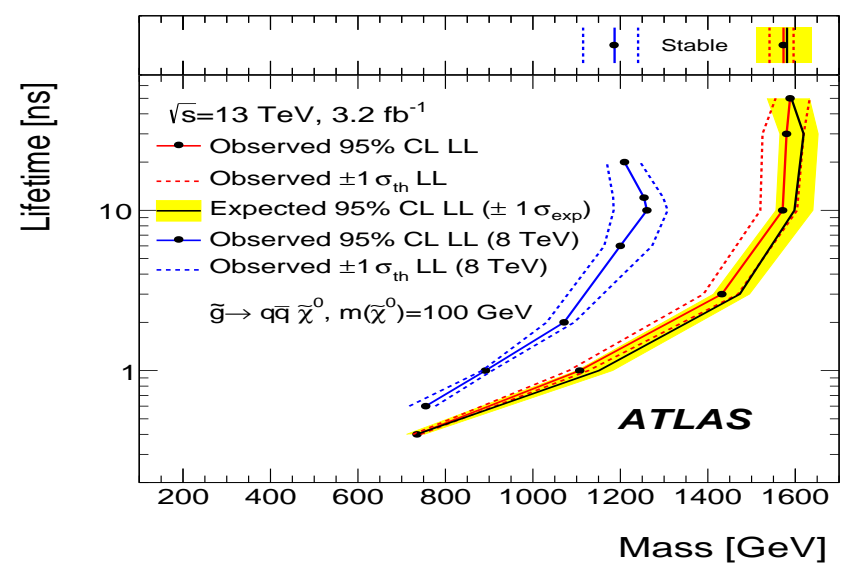

Figure 4. Excluded range of lifetimes as a function of gluino R-hadron mass [14]. The expected lower limit (LL), with its experimental $\pm 1 \sigma$ band, is given with respect to the nominal theoretical cross-section. The observed 95\% LL obtained at $\sqrt{s}=8 \mathrm{TeV}$ is also shown for comparison.

\section{Summary}

One noticeable upgrade from Run-1 to Run-2 in the ATLAS experiment is the introduction of the new pixel detector IBL located near the beam pipe as the extra innermost pixel layer. The tracking and vertex reconstruction are significantly improved and good performance is expected in high level object such a $b$-quark jet tagging, in turn, it leads the better physics results. The note summarizes what is the impact on the IBL detector to the physics results especially focusing on the 
analyses using the $b$-quark jets throughout 2016 summer physics program. Three distinct topics are discussed; high signal efficiency (SUSY multi $b$-quark jet topology), large background rejection (the Standard Model $t \bar{t} H$ ) and the other application of the lifetime measurement (SUSY long-lived particles). For all aspects, it is revealed that the physics performance is largely improved over the expectation without IBL.

\section{References}

[1] ATLAS Collaboration, The ATLAS Experiment at the CERN Large Hadron Collider, JINST 3 (2008) S08003.

[2] All ATLAS results are found in https://twiki.cern.ch/twiki/bin/view/AtlasPublic/Summer2016-13TeV.

[3] ATLAS Collaboration, ATLAS Insertable B-Layer Technical Design Report, CERN-LHCC-2010-013; ATLAS-TDR-19.

[4] ATLAS Collaboration, ATLAS pixel detector : Technical Design Report, ATLAS-TDR-11; CERN-LHCC-98-013.

[5] ATLAS Collaboration, Impact parameter resolution Run-1 vs. Run-2, ITDR-2015-007 (2015).

[6] ATLAS Collaboration, Expected performance of the ATLAS b-tagging algorithms in Run-2, ATL-PHYS-PUB-2015-022.

[7] ATLAS Collaboration, A neural network clustering algorithm for the ATLAS silicon pixel detector, JINST 9 (2014) P09009.

[8] ATLAS Collaboration, Search for pair production of gluinos decaying via top or bottom squarks in events with $b$-jets and large missing transverse momentum in $p p$ collisions at $\sqrt{s}=13 \mathrm{TeV}$ with the ATLAS detector, ATLAS-CONF-2016-052.

[9] ATLAS Collaboration, Search for strong production of supersymmetric particles in final states with missing transverse momentum and at least three b-jets at $\sqrt{s}=8 \mathrm{TeV}$ proton-proton collisions with the ATLAS detector, JHEP 10 (2014) 024; arXiv: 1407.0600.

[10] W.J. Stirling, private communication. See reference, http://www.hep.ph.ic.ac.uk/ wstirlin/plots/plots.html.

[11] ATLAS Collaboration, Expected sensitivity studies for gluino and squark searches using the early LHC 13 TeV Run-2 dataset with the ATLAS experiment, ATL-PHYS-PUB-2015-005.

[12] ATLAS Collaboration, Search for the Standard Model Higgs boson produced in association with top quarks and decaying into a b $\bar{b}$ pair in pp collisions at $\sqrt{s}=13 \mathrm{TeV}$ with the ATLAS detector, ATLAS-CONF-2016-080.

[13] ATLAS Collaboration, Search for the Standard Model Higgs boson produced in association with top quarks and decaying into bb in pp collisions at $\sqrt{s}=8 \mathrm{TeV}$ with the ATLAS detector, Eur. Phys. J. C75 (2015) 349; arXiv:1503.05066.

[14] ATLAS Collaboration, Search for metastable heavy charged particles with large ionization energy

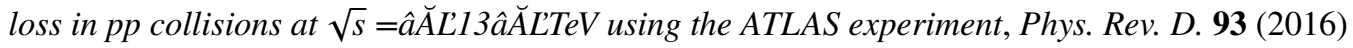
112015; arXiv:1604.04520.

[15] ATLAS Collaboration, Search for metastable heavy charged particles with large ionisation energy loss in pp collisions at $\sqrt{s}=8 \mathrm{TeV}$ using the ATLAS experiment, Eur. Phys. J. C75 (2015) 407; arXiv:1506.05332. 